\title{
REVIEW \\ Beliefs relating to recurrence of heterotopic ossification following excision in patients with spinal cord injury: a review
}

\author{
F Genêt ${ }^{1,2,3}$, A Ruet ${ }^{1,4}$, W Almangour ${ }^{1}$, L Gatin $^{5}$, P Denormandie ${ }^{2,5}$ and A Schnitzler ${ }^{1,4}$
}

Study design: Review of the literature.

Objectives: It is widely believed that the timing of surgery and the size of the initial Neurological Heterotopic Ossification (NHO) affect the recurrence risk of $\mathrm{NHO}$ after $\mathrm{SCl}$. A large number of studies were published in the 80 s and the 90s, mostly of poor quality despite the fact that they were carried out by experienced surgical teams. The aim of this study was to suggest recommendations relating to the timing of excision of heterotopic ossification after $\mathrm{SCl}$ following the analysis of a recent review of the literature.

Setting: France.

Methods: A systematic literature search was performed in the PubMed Embase from January 2002 until June 2014 using the MESH headings 'spinal cord injury', 'paraplegia', 'heterotopic ossification' and 'surgery'. Results were compared with results from epidemiological studies based on the BANKHO database (patients who underwent surgery for troublesome HO after central neurological system (CNS) lesions in our center (357 patients, 539 surgeries)).

Results: Few studies were found in the literature, results were sometimes contradictory and practices heterogeneous. Results from the BANKHO database showed that troublesome recurrence of NHO was not associated with 'early' surgery (before 6 months), and no association was found between recurrence and the size of the $\mathrm{NHO}$ around the joint (Brooker status).

Conclusion: We suggest that surgical excision of the NHO should be carried out when it begins to be troublesome, as soon as comorbid factors are under control and the $\mathrm{HO}$ is sufficiently constituted for excision.

Spinal Cord (2015) 53, 340-344; doi:10.1038/sc.2015.20; published online 17 February 2015

\section{INTRODUCTION}

Following spinal cord injury (SCI), between $20 \%$ and $30 \%$ of patients develop ectopic lamellar bone tissue around certain joints. ${ }^{1}$ This is termed Neurological Heterotopic Ossification $(\mathrm{NHO}) .^{2-4}$

NHO causes pain, decreased range of motion (ROM) until complete ankylosis occurs, functional disabilities such as difficulty sitting, standing, walking, eating, dressing and so on, depending on the location of the $\mathrm{NHO}^{1,3-7}$ and may cause nerve and vessel compression. $^{7-9}$ The timing of its occurrence depends on the time of the diagnosis (whether based on clinical diagnosis or imagery), but NHO usually occurs between 2 weeks and 3 months after the central neurological system (CNS) lesion, sometimes later., ${ }^{1,40}$ The etiopathogenesis is still poorly understood. With regard to curative treatments, medical interventions have limited effectiveness, and there are currently no guidelines for the management of this troublesome side effect despite the publication of six quality reviews in the past 5 years. $1,3,7,11-13$ Since the beginning of the twentieth century, the only effective treatment of NHO that has been found is surgical excision. ${ }^{1,2,5,7}$ The literature presents globally consistent results of the first surgical excisions with an increase in ROM. ${ }^{1,3,14,15}$ Some studies report functional improvements (ability to sit, to stand up, to walk, to eat, to access to the perinea or axillary area), reduction in pain (that is, nerve decompression) or improvements in bedsores. ${ }^{4,7,12}$

One of the main complications after surgical excision is recurrence. This is frequently observed on X-ray as an ossification process occurring around the joint after the excision. In clinical practice, this must be taken into account, particularly when it is troublesome, that is, when it is painful, or impacts a ROM and functional ability. ${ }^{16}$ From the 70 s to the end of the 90 s, the rate of recurrence diagnosed by roentgenogram for patients with SCI was estimated to be between $82 \%$ and $100 \%$ of cases, and the rate of recurrence diagnosed by clinical symptoms was estimated between $17 \%$ and $58 \%{ }^{4,10,16}$ Some beliefs regarding the risk of postsurgical recurrence of $\mathrm{NHO}$ have influenced clinical practice for several decades. Several teams have suggested that the association of radiation therapy, passive ROM therapy and/or bisphosphonate injections prior to surgical excision could reduce the risk of recurrence, ${ }^{16-20}$ but no real studies have been carried out to evaluate these methods. ${ }^{4}$ It is still believed that the NHO must be mature prior to surgery to reduce the risk of recurrence (no surgery before 1 year) despite the fact that earlier removal would be beneficial for other factors. ${ }^{7}$ It is also thought that the extent of the initial NHO affects recurrence. ${ }^{7}$ These questions are regularly raised;

${ }^{1}$ Department of Physical Medicine and Rehabilitation, Hôpital Raymond Poincaré, APHP, CIC-IT 1429, Garches, France; ${ }^{2}$ Université Versailles Saint Quentin en Yvelines, "End: icap" U1179 INSERM, UFR des Sciences de la Santé-Simone Veil, Montigny le Bretonneux, France; '3Service de Médecine Physique et de Réadaptation, Service de Santé des Armées, Hôpital d'Instruction des Armées Percy, Clamart, France; ${ }^{4}$ University of Versailles Saint Quentin en Yvelines, HANDI-ReSP (EA4047), UFR des Sciences de la SantéSimone Veil, Montigny le Bretonneux, France and ${ }^{5}$ Service de Chirurgie Orthopédique, Hôpital Raymond Poincaré, APHP, Garches, France

Correspondence: Dr F Genêt, Department of Physical Medicine and Rehabilitation, Raymond Poincare Hospital, 104 bd Raymond Poincaré, Garches 92380 , France. E-mail: francois.genet@rpc.aphp.fr

Received 5 September 2014; revised 15 December 2014; accepted 12 January 2015; published online 17 February 2015 
however, according to our clinical experience, there is no impact of timing of surgery or extent of the $\mathrm{HO}$ on recurrence. The aim of this study was therefore to investigate the validity of these beliefs by reviewing studies in the literature along with the results of epidemiological studies from a database that included data from patients who underwent surgery for troublesome $\mathrm{HO}$ after CNS lesions in our center (BANKHO database). Two questions were posed: (1) Does the timing of the surgical intervention affect the risk of recurrence of troublesome HO after SCI? and (2) Does the extent of the HO affect the risk of recurrence of troublesome $\mathrm{HO}$ after SCI?

\section{MATERIALS AND METHODS}

\section{Systematic literature search}

A systematic literature search was performed in the PubMed Embase between January 2002 and June 2014. This period was chosen because during this time there was little change in intensive care practices and surgical techniques (both could affect $\mathrm{HO}$ development and postoperative recurrence), thus allowing comparisons between studies. Moreover, a profusion of studies on surgery for $\mathrm{HO}$ and risk of recurrence was published during the 80 s and the beginning of the $90 \mathrm{~s}$. Interest in this topic then decreased before several teams published studies creating some controversy regarding the timing of surgical removal of NHOs at the beginning of the twenty-first century. In 2001, Banovac et al..$^{21}$ found a reduction in the prevalence of $\mathrm{HO}$ after SCI with the use of prophylactic nonsteroidal anti-inflammatory drugs in a randomized double-blind controlled study. This was the first publication to show that a medical treatment could have an effect on this symptom. In 2002, Van Kuijk et al. ${ }^{4}$ reported in an important review that the timing of surgical intervention did not appear to affect risk of recurrence, in contrast with the extent of the HO. The review was based on the publications by Garland et al. ${ }^{10,22,23}$ (during the $80 \mathrm{~s}$ and beginning of the 90s) and two studies on small samples of patients (Mc Auliffe et al. ${ }^{20}$ and Freebourn et al. ${ }^{18}$ ).

For the literature search, the MESH headings 'spinal cord injury' and 'heterotopic ossification' were used, and the search was restricted to studies written in English or French. The titles and abstracts of the studies found were screened in order to select those that reported on the timing of $\mathrm{HO}$ excision and recurrence in patients with spinal cord lesions (other etiologies were excluded, that is, traumatic brain injury, stroke, orthopedic conditions, total hip arthroplasty and burns). Subsequently, the full texts of the selected studies were independently screened by two authors (FG and AS) for eligibility. In the $80 \mathrm{~s}$ and $90 \mathrm{~s}$, a profusion of studies was carried out on this topic and they have influenced current beliefs. It was thus considered appropriate to provide a short summary of the conclusions of the principle articles as a historical background to each of the two questions discussed.

\section{Inclusion and exclusion criteria}

Inclusion criteria were studies of patients with SCI who underwent surgery for troublesome heterotopic ossification. All articles (studies and reviews) had to describe the following essential outcomes relating to the two questions investigated in this study in order to be included: etiology of the neurological lesion, time from diagnosis to surgery, postsurgical complications, recurrence of $\mathrm{HO}$ and the time from surgery to recurrence. Patients had to be followed up for at least 3 months. To answer question 2 (effect of the extent of the HO on recurrence risk), studies also had to report the results of imagery (Xray and/or computed tomography scan) and the clinical assessment.

No studies that used statistical analysis to evaluate the impact of the timing of surgery on recurrence were found, therefore articles that discussed the experience of surgical teams were accepted for inclusion.
Articles with no available abstract were not considered.

\section{Assessment of study quality}

As stated above, although the studies selected were carried out by surgical teams experienced in $\mathrm{HO}$ excision, the quality of studies was poor. There were no randomized controlled trials, and the scales used were frequently not validated for this purpose. This is mainly because the diagnosis of $\mathrm{HO}$ is always delayed relative to its occurrence, the incidence is relatively low, sample sizes are small and the etiopathogenesis is still poorly understood, making early diagnosis difficult.

\section{BANKHO database}

A database named 'BANKHO' has been developed since 1993 in our center. It includes data from patients who have undergone surgery for troublesome HO after CNS lesions. ${ }^{5}$ In November 2009, the database contained data from 357 patients, including 539 first-time interventions for HO (129 for multiple sites). All the surgical interventions in the database were performed by the same surgeon (PD).

In 2009, an epidemiological study based on the database was carried out in order to address the two questions posed above. The results showed that most of the HOs, which required surgery, occurred after traumatic brain injury (199 patients $(55.7 \%))$, followed by spinal cord injury $(86(24.0 \%))$, stroke $(42(11.8 \%))$ and cerebral anoxia $(30$ $(8.6 \%))$. The hip was the primary site of HO $(328(60.9 \%))$, followed by the elbow $(115(21.3 \%))$, knee $(77(14.3 \%))$ and shoulder (19 $(3.5 \%))$. Symptomatic recurrences requiring further surgical intervention occurred in 31 cases $(5.8 \%$ (31/539); 95\% confidence interval: $3.8-7.8 \%$; 27 patients).

\section{RESULTS}

\section{Included studies}

Twelve from January 2002 to June 2014 (468 were identified when the year of the start of the search was not limited, 70 of which were included) were included.

1. Impact of the timing of surgery on the risk of recurrence: 12 articles published since 2002 were accepted: 8 reviews $^{2-4,7,13,24-26}$ and 4 studies $^{5,27-29}$ out of the 30 published.

2. Impact of the size of the $\mathrm{HO}$ on the risk of recurrence: 4 articles published since 2002 were accepted: 3 reviews ${ }^{2,4,24}$ and 1 study $^{28}$ out of the 30 published.

Does the timing of surgical intervention affect the risk of recurrence of troublesome $\mathrm{HO}$ after SCI?

Historical approach. During the 90s, following the experience and publications of Garland et al., ${ }^{22}$ several teams agreed to wait for at least the arbitrary 12 months after CNS lesion before considering surgery. The main reason was to ensure that maximal neurological recovery had occurred if the aim of surgery was to improve function, as well as to avoid recurrence (by waiting for neurological stabilization and mainly stabilization of spasticity). Despite respecting this delay, Garland et al. ${ }^{10}$ reported a $36 \%$ recurrence rate of significant, clinically mature NHO after SCI (based on scintigraphy). They also reported that normal bone scans, alkaline phosphatase levels and the mature roentgenographic appearance of $\mathrm{HO}$ were not reliable predictors of recurrence. ${ }^{10}$ On the basis of these results, some teams concluded that there was no relationship between the timing of surgical intervention relative to the onset of the spinal lesion and risk of recurrence. ${ }^{30}$ 
Since 2002. It is currently widely accepted that the HO must reach maturity before surgery is carried out in order to reduce the risk of recurrence. ${ }^{7,24,25}$ However, some teams have suggested that this is not founded ${ }^{3}$ and that studies evaluating the optimal timing are necessary. ${ }^{4}$ One major problem is the difficulty in determining whether the NHO has reached maturity. ${ }^{13}$ Numerous methods have been tested (roentgenogram, computed tomography scan, biological, three-phase-bone scan and so on) with controversial results and samples, which were quite often heterogeneous. ${ }^{4}$ Moreover, these studies frequently lack statistical analysis. Shehab et al. ${ }^{25}$ discussed the surgeon's predicament: waiting for NHO maturity to avoid recurrence exposes the patient to prolonged pain and loss of joint motion, both of which may reduce functional ability and probably neurological recovery, as well as increasing the risk of postoperative complications such as hematoma or fracture. Because there is a lack of studies regarding the most effective time to perform the resection, physicians must attempt to balance the risk of recurrence against that of potential complications. ${ }^{7}$ According to Vanden Bossche et al., ${ }^{2}$ the functional and neurological prognosis is the greatest priority to determine the timing of surgery, over the risk of recurrence. Unfortunately, whatever the timing of surgery (from less to more than 18 months after onset of the CNS lesion), most of the studies on this topic have reported very disparate rates of $\mathrm{NHO}$ recurrence (from none to $92 \%$ roentgenogram recurrence). ${ }^{27,29}$ One point that arises from these studies is that, even if operative delays and follow-up are very heterogeneous in each study, roentgenographic recurrence (without symptoms) is largely more frequent compared with clinical recurrence (with symptoms). Consequently, it is not clear whether the timing of surgery has an impact on clinical recurrence. ${ }^{7,26}$

The 'BANKHO' database. There were no recurrences of HO following the surgical interventions, which were performed during the first year after the SCI (181/539 interventions). ${ }^{5}$ For the patients for whom recurrence occurred (all had surgery after 1 year), it was not associated with etiology, sex, age at time of CNS lesion, multisite NHO or 'early' surgery (before 6 months). Recurrence was evaluated in the total sample (539 surgeries), ${ }^{5}$ as well as in sub-groups comprising traumatic brain injury and SCI patients (95 interventions). ${ }^{28}$

\section{Does the extent of the $\mathrm{HO}$ affect the risk of recurrence of troublesome $\mathrm{HO}$ after CNS lesion?}

Historical approach. The second factor, which is believed to have an impact on recurrence of troublesome $\mathrm{HO}$ after CNS lesion, is the extent and the number of HOs for each patient. ${ }^{10}$ The most commonly used classification to determine the volume of the NHO is that of Brooker, who developed a method in 1973 to classify the degree of ectopic bone formation around the hip following total hip arthroplasty. ${ }^{31}$ This classification is useful in that it can be based on a single anteroposterior X-ray of the hip. This measurement appeared to correlate with global hip function. ${ }^{32}$ Garland et al. ${ }^{10}$ reported an association between the risk of recurrence and the roentogenographic grade (maturity and extent) of the NHO in SCI patients. Experienced teams have suggested that Brooker's classification should be systematically used, although this is based on a study without statistical analysis. $^{16}$

Since 2002. There is currently no consensus for roentgenogram classification of NHO, ${ }^{2,24}$ Several teams have tried to find a link between the risk of recurrence and the preoperative extent of the $\mathrm{NHO}$; however, results are based on clinical observations and are consequently only descriptive. ${ }^{4,24}$ The Brooker classification remains the most used for the classification of NHO, although its validity has never been evaluated in patients with neurological lesions and joints other than the hip. ${ }^{33}$ Furthermore, for HOs of orthopedic etiology, Mavrogenis et al. ${ }^{24}$ highlight that the disadvantage of this scale is that it does not indicate the anatomical compartment in which the HOs are situated. Consequently, there is no correlation between the score and the extent of the HOs in each compartment, the scale cannot be used to determine the limitations expected from surgery and it does not fully help the surgeon to determine the surgical approach and the prognosis. ${ }^{24}$ Moreover, the results of the present study showed, similarly to a study of $\mathrm{HO}$ of orthopedic etiology, ${ }^{34}$ that the Brooker measurement gave pessimistic expectations of hip ROM. Indeed, HOs around the hip, which were classified III or IV, were not clinically ankylosed. ${ }^{24}$

'BANKHO' database. We carried out a case-control study using the BANKHO database. ${ }^{28}$ No significant relationship was found between recurrence and the location around the joint or the Brooker status, even when all the matching factors were included in the analysis.

\section{DISCUSSION}

\section{Main results}

During the past decade, few studies have assessed whether the timing of surgery and the size of the NHO after SCI are risk factors for recurrence after surgical excision. Recent reviews have provided opinions on this subject, but these are based on the results of older studies. Moreover, few articles have been specifically related to SCI, and thus the results and conclusions are not specific to different etiologies. The lack of homogeneity of the etiology of patients included is a methodological weakness of previous studies. However, recent studies appear to confirm that the timing of surgery and the extent of the $\mathrm{HO}$ are not risk factors for recurrence after surgical excision in patients with SCI.

\section{Why is surgery still the only curative treatment for NHO?}

Surgery is effective. As discussed in the introduction, the literature presents globally consistent results of the first surgical excisions of $\mathrm{HO}$ with an increase in ROM and sometimes a functional improvement, a reduction in pain or an improvement in bedsores. Moreover, a prolonged delay before excision leads to a negative cascade of events: risk of ankylosis, intra-articular lesions, bone loss in the femoral head and increased risk of fracture during or after surgery. $4,6,16,33$

Medical treatments for prevention and cure are still insufficient. Medical treatments for prevention and cure of $\mathrm{HO}$ are not very effective, mostly because of the lateness of the diagnosis along with the lack of well-established risk factors. This has been well discussed in recent reviews on the subject. ${ }^{1,3,7,11-13}$ Diagnosis is particularly difficult in the early phase of HO development because of the severity of the patient's general condition. Indeed, the motor, sensory and cognitive impairments after CNS lesions, the nonspecific symptoms of $\mathrm{HO}$ and the poor correlation between clinical symptoms and roentgenogram signs, which are moreover delayed for several weeks, all reduce the sensitivity of an early diagnosis. Biological diagnosis is not particularly useful mainly because the results are not specific.,10 There are several methods of prevention of HO, but all have limitations. Studies carried out in patients with SCI provide strong support for the use of nonsteroidal anti-inflammatory drugs; ${ }^{21,35}$ however, their use is limited by their side effects, which include gastric problems and delayed bone repair (undesirable in a posttrauma population). ${ }^{11,36,37}$ Pulsed electromagnetic fields also appear effective as prevention. ${ }^{38}$ 
Bisphosphonates (etidronate) seem to delay $\mathrm{HO}$ development in traumatic brain injury and SCI patients if given very early and for a prolonged period ( 6 months). ${ }^{23,35,39-41}$ Finally, radiation therapy may be used as prevention ${ }^{42}$ but has not yet been well evaluated in patients with CNS lesions. The young age of the patients affected and the potential side effects of radiation also justify further studies. The risk factors for $\mathrm{HO}$ do not seem to depend on the patient's medical symptoms but mainly on the severity of his/her condition during the acute phase and the duration of immobility before regular mobilization begins. ${ }^{43-46}$

No animal model of acquired HO after CNS lesion. If an animal model that develops acquired $\mathrm{HO}$ after CNS lesion existed, each of the retrospective factors suspected by clinicians to affect $\mathrm{HO}$ development could be assessed. This would increase understanding of their impact on the early process of development of heterotopic ossification. Moreover, biological factors involved in $\mathrm{HO}$ formation as a function of the type of CNS lesion could be sought. Unfortunately, such a model does not currently exist and researchers have used transgenic mice, which overexpress bone morphogenetic protein 4 , implantation of matrigel and scaffolds to induce $\mathrm{HO}$ and intramuscular injection of transduced cells by adenovirus that over express bone morphogenetic protein $2 .^{47-49}$

Finally, as the pathophysiological process is still misunderstood, diagnosis is delayed, risk factors are cofounding, preventive or curative medical treatments are hazardous or not well assessed and, as there is no specific animal model to enhance our knowledge about the induction of such a troublesome condition, the only curative treatment remains mechanical excision.

\section{Recurrences}

Another problem of definition and clinical relevance. Recurrence is one of the three main complications of HO excision, along with sepsis and hematoma. The postoperative rate of recurrence is rarely assessed for three main reasons. First of all, there is no accepted definition of recurrence. ${ }^{4,7,22,26}$ It may be a clinical finding without any impact on the patient's function (for example, decrease in ROM without any impact on the patient's life considering his/her disabilities) or it may be a troublesome clinical finding. ${ }^{16}$ It is true that the rates of recurrence found in the studies by our team are quite low compared with results in the literature (from $17 \%$ to $58 \%{ }^{10,16,41,50,51}$ ). The first reason is that this rate includes only the patients (with neurological lesions) who required surgical revision of their $\mathrm{HO}$ removal. A large proportion of recurrences (with roentographic or clinical signs) were not considered as 'real recurrence' because they did not impact on the patient's function (not treated because no impact). We propose that revision of $\mathrm{HO}$ recurrence should be considered when it is uncomfortable or painful for the patient or if the loss of ROM affects function. Second, surgical techniques, perioperative and postoperative care and patient follow-up are not standardized, which may have an impact on the rate of recurrence. For example, most of the studies include early rehabilitation after surgical excision, ${ }^{7}$ but because local inflammation (mainly in the muscles around the joint) seems to have a strong impact on $\mathrm{HO}$ formation, ${ }^{21}$ we generally delay the mobilization of the limb until 1 week after surgery and prescribe a preventive anti-inflammatory treatment (nonsteroidal anti-inflammatory drug).

\section{Limits}

The ambispective analysis could be a limit of the studies based on the BANKHO database. However, in clinical practice, it is difficult to carry out prospective studies on $\mathrm{HO}$ because the rate of occurrence in the intensive care unit is low and the diagnosis is delayed. The inclusion of data in the database was carried out over a relatively long period of time; however, surgical and medical management has not changed in our center since 1993, and thus we do not believe that this could affect the results. The fact that the data all come from a single center with one surgeon carrying out all the interventions may have led to some bias. However, these studies were among the first to assess the impact of the timing of surgery on recurrence of $\mathrm{HO}$ using an epidemiological approach.

\section{CONCLUSIONS}

Results in the literature along with results from studies using the BANKHO database suggest that the preoperative extent of the $\mathrm{HO}$ around the joint is not related to the risk of recurrence following excision. These results lead us to suggest that surgical excision of $\mathrm{HO}$ should be carried out as soon as it becomes troublesome, comorbid factors are under control and the HO is sufficiently constituted for excision. Moreover, early excision should allow patients to reach their functional potential as they will not be impeded by restrictions in passive joint motion. It would also improve comfort and reduce pain. Delaying surgery and allowing ankylosis to occur increase the risk of bone loss beneath the $\mathrm{HO}$ and also increase the risk of fracture during or after surgery.

\section{CONFLICT OF INTEREST}

The authors declare no conflict of interest.

\section{ACKNOWLEDGEMENTS}

We thank Mrs Erica Vlachos (CRA) for the management of the database.

1 Teasell RW, Mehta S, Aubut JL, Ashe MC, Sequeira K, Macaluso S et al. A systematic review of the therapeutic interventions for heterotopic ossification after spinal cord injury. Spinal Cord 2010; 48: 512-521.

2 Vanden Bossche L, Vanderstraeten G. Heterotopic ossification: a review. J Rehabil Med 2005; 37: 129-136.

3 Aubut J-AL, Mehta S, Cullen N, Teasell RW, ERABI Group, Scire Research Team. A comparison of heterotopic ossification treatment within the traumatic brain and spinal cord injured population: An evidence based systematic review. NeuroRehabilitation 2011; 28: 151-160.

4 Van Kuijk AA, Geurts ACH, van Kuppevelt HJM. Neurogenic heterotopic ossification in spinal cord injury. Spinal Cord 2002; 40: 313-326.

5 Genêt F, Jourdan C, Schnitzler A, Lautridou C, Guillemot D, Judet T et al. Troublesome heterotopic ossification after central nervous system damage: a survey of 570 surgeries. PLOS ONE 2011; 6: e16632.

6 Genet F, Marmorat J-L, Lautridou C, Schnitzler A, Mailhan L, Denormandie P. Impact of late surgical intervention on heterotopic ossification of the hip after traumatic neurological injury. J Bone Joint Surg Br 2009; 91: 1493-1498.

7 Cipriano CA, Pill SG, Keenan MA. Heterotopic ossification following traumatic brain injury and spinal cord injury. J Am Acad Orthop Surg 2009; 17: 689-697.

8 Cullen N, Bayley M, Bayona N, Hilditch M, Aubut J, Erabi Group. Management of heterotopic ossification and venous thromboembolism following acquired brain injury. Brain Inj 2007; 21: 215-230.

9 Abayev B, Ha E, Cruise C. A sciatic nerve lesion secondary to compression by a heterotopic ossification in the hip and thigh region-an electrodiagnostic approach. Neurologist 2005; 11: 184-186.

10 Garland DE, Orwin JF. Resection of heterotopic ossification in patients with spinal cord injuries. Clin Orthop 1989; 242: 169-176.

11 Baird EO, Kang QK. Prophylaxis of heterotopic ossification-an updated review. J Orthop Surg 2009; 4: 12.

12 Sullivan MP, Torres SJ, Mehta S, Ahn J. Heterotopic ossification after central nervous system trauma: a current review. Bone Jt Res 2013; 2: 51-57.

13 Sakellariou VI, Grigoriou E, Mavrogenis AF, Soucacos PN, Papagelopoulos PJ. Heterotopic ossification following traumatic brain injury and spinal cord injury: insight into the etiology and pathophysiology. J Musculoskelet Neuronal Interact 2012; 12: 230-240.

14 Mitsionis GI, Lykissas MG, Kalos N, Paschos N, Beris AE, Georgoulis AD et al. Functional outcome after excision of heterotopic ossification about the knee in ICU patients. Int Orthop 2009; 33: 1619-1625. 
15 Ellerin BE, Helfet D, Parikh S, Hotchkiss RN, Levin N, Nisce L et al. Current therapy in the management of heterotopic ossification of the elbow: a review with case studies. Am J Phys Med Rehabil 1999; 78: 259-271.

16 Stover SL, Niemann KM, Tulloss JR. Experience with surgical resection of heterotopic bone in spinal cord injury patients. Clin Orthop 1991; 263: 71-77.

17 Meiners T, Abel R, Böhm V, Gerner HJ. Resection of heterotopic ossification of the hip in spinal cord injured patients. Spinal Cord 1997; 35: 443-445.

18 Freebourn TM, Barber DB, Able AC. The treatment of immature heterotopic ossification in spinal cord injury with combination surgery, radiation therapy and NSAID. Spinal Cord 1999; 37: 50-53.

19 Biering-Sørensen F, Tøndevold E. Indomethacin and disodium etidronate for the prevention of recurrence of heterotopic ossification after surgical resection. Two case reports. Paraplegia 1993; 31: 513-515.

20 McAuliffe JA, Wolfson AH. Early excision of heterotopic ossification about the elbow followed by radiation therapy. J Bone Joint Surg Am 1997; 79: 749-755.

21 Banovac K, Williams JM, Patrick LD, Haniff YM. Prevention of heterotopic ossification after spinal cord injury with indomethacin. Spinal Cord 2001; 39: 370-374.

22 Garland DE. A clinical perspective on common forms of acquired heterotopic ossification. Clin Orthop 1991; 263: 13-29.

23 Garland DE, Alday B, Venos KG, Vogt JC. Diphosphonate treatment for heterotopic ossification in spinal cord injury patients. Clin Orthop. 1983; 176: 197-200.

24 Mavrogenis AF, Guerra G, Staals EL, Bianchi G, Ruggieri P. A classification method for neurogenic heterotopic ossification of the hip. J Orthop Traumatol 2012; 13 69-78.

25 Shehab D, Elgazzar AH, Collier BD. Heterotopic ossification. J Nucl Med 2002; 43 : 346-353.

26 Kaplan FS, Glaser DL, Hebela N, Shore EM. Heterotopic ossification. J Am Acad Orthop Surg 2004; 12: 116-125.

27 Carlier RY, Safa DML, Parva P, Mompoint D, Judet T, Denormandie P et al. Ankylosing neurogenic myositis ossificans of the hip. An enhanced volumetric CT study. J Bone Joint Surg Br 2005; 87: 301-305.

28 Genêt F, Jourdan C, Lautridou C, Chehensse C, Minooee K, Denormandie P et al. The impact of preoperative hip heterotopic ossification extent on recurrence in patients with head and spinal cord injury: a case control study. PLOS ONE 2011; 6: e23129.

29 Fuller DA, Mark A, Keenan MAE. Excision of heterotopic ossification from the knee: a functional outcome study. Clin Orthop 2005; 438: 197-203.

30 Stover SL, Niemann KM, Tulloss JR. Experience with surgical resection of heterotopic bone in spinal cord injury patients. Clin Orthop Relat Res 1991, 71-77.

31 Brooker AF, Bowerman JW, Robinson RA, Riley LH. Ectopic ossification following total hip replacement. Incidence and a method of classification. J Bone Joint Surg Am 1973; 55: 1629-1632.

32 Wright JG, Moran E, Bogoch E. Reliability and validity of the grading of heterotopic ossification. J Arthroplasty 1994; 9: 549-553.

33 Melamed E, Robinson D, Halperin N, Wallach N, Keren O, Groswasser Z. Brain injuryrelated heterotopic bone formation: treatment strategy and results. Am J Phys Med Rehabil 2002; 81: 670-674.
34 Schuh A, Zeiler G. [The modified Brooker classification for evaluation of heterotopic ossifications in total hip replacement]. Zentralblatt Für Chir 2005; 130 293-296.

35 Banovac K, Gonzalez F, Wade N, Bowker JJ. Intravenous disodium etidronate therapy in spinal cord injury patients with heterotopic ossification. Paraplegia 1993; 31 660-666.

36 Burd TA, Hughes MS, Anglen JO. Heterotopic ossification prophylaxis with indomethacin increases the risk of long-bone nonunion. J Bone Joint Surg Br 2003; 85: 700-705.

37 Dahners LE, Mullis BH. Effects of nonsteroidal anti-inflammatory drugs on bone formation and soft-tissue healing. J Am Acad Orthop Surg 2004; 12: 139-143.

38 Durović A, Miljković D, Brdareski Z, Plavsić A, Jevtić M. Pulse low-intensity electromagnetic field as prophylaxis of heterotopic ossification in patients with traumatic spinal cord injury. Vojnosanit Preg/ 2009; 66: 22-28.

39 Banovac K. The effect of etidronate on late development of heterotopic ossification after spinal cord injury. J Spinal Cord Med 2000; 23: 40-44.

40 Banovac K, Gonzalez F. Evaluation and management of heterotopic ossification in patients with spinal cord injury. Spinal Cord 1997; 35: 158-162.

41 Stover SL, Niemann KM, Miller JM. Disodium etidronate in the prevention of postoperative recurrence of heterotopic ossification in spinal-cord injury patients. J Bone Joint Surg Am 1976; 58: 683-688.

42 Cipriano C, Pill SG, Rosenstock J, Keenan MA. Radiation therapy for preventing recurrence of neurogenic heterotopic ossification. Orthopedics [Internet] 2009; 32. Disponible sur http://www.ncbi.nlm.nih.gov/entrez/query.fcgi? cmd = Retrieve\&db = PubMed\&dopt $=$ Citation\&list_uids $=19750999$.

43 Silver JR. Heterotopic ossification. A clinical study of its possible relationship to trauma. Paraplegia 1969; 7: 220-230.

44 Silver JR. A systematic review of the therapeutic interventions for heterotopic ossification after spinal cord injury. Spinal Cord 2011; 49: 482 (author reply 484).

45 Daud O, Sett P, Burr RG, Silver JR. The relationship of heterotopic ossification to passive movements in paraplegic patients. Disabil Rehabil 1993; 15: $114-118$.

46 Izumi K. Study of ectopic bone formation in experimental spinal cord injured rabbits. Paraplegia 1983; 21: 351-363.

47 Kan L, Hu M, Gomes WA, Kessler JA. Transgenic mice overexpressing BMP4 develop a fibrodysplasia ossificans progressiva (FOP)-like phenotype. Am J Pathol 2004; 165: 1107-1115.

48 Kan L, Kessler JA. Animal models of typical heterotopic ossification. J Biomed Biotechnol 2011; 2011: 309287.

49 Lazard ZW, Heggeness MH, Hipp JA, Sonnet C, Fuentes AS, Nistal RP et al. Cell-based gene therapy for repair of critical size defects in the rat fibula. J Cell Biochem 2011; 112: 1563-1571.

50 Hsu JD, Sakimura I, Stauffer ES. Heterotopic ossification around the hip joint in spinal cord injured patients. Clin Orthop 1975, 165-169.

51 Wharton GW, Morgan TH. Ankylosis in the paralyzed patient. J Bone Joint Surg Am 1970; 52: 105-112. 